\title{
Neuroscience and education: prime time to build the bridge
}

\author{
Mariano Sigman $^{1,2}$, Marcela Peña ${ }^{3}$, Andrea P Goldin ${ }^{1,2} \&$ Sidarta Ribeiro ${ }^{4}$
}

As neuroscience gains social traction and entices media attention, the notion that education has much to benefit from brain research becomes increasingly popular. However, it has been argued that the fundamental bridge toward education is cognitive psychology, not neuroscience. We discuss four specific cases in which neuroscience synergizes with other disciplines to serve education, ranging from very general physiological aspects of human learning such as nutrition, exercise and sleep, to brain architectures that shape the way we acquire language and reading, and neuroscience tools that increasingly allow the early detection of cognitive deficits, especially in preverbal infants. Neuroscience methods, tools and theoretical frameworks have broadened our understanding of the mind in a way that is highly relevant to educational practice. Although the bridge's cement is still fresh, we argue why it is prime time to march over it.

Schooling also is becoming more and more necessary to an understanding of adult problems in this society; and a certain amount of wealth, of freedom from economic pressure, may be quite necessary to full intellectual development. The fact is, however, that we know almost nothing specific about the matter. The country may be full of potential geniuses, for all we know, and it should be a pressing concern for psychology to discover the conditions that will develop whatever potentialities a child may have.

-Donald Hebb, The Organization of Behavior (1949).

Around the globe, over 2 billion children attend school daily, in what constitutes the largest learning experiment ever attempted. For this reason, and because rising education inequality is among the gravest of the world's problems, the time for evidence-based education has arrived, with ambitious goals for pedagogical experiments that may lead to breakthrough discoveries and translate to effective social improvement.

Despite the obvious fact that education is constrained by the architecture and functioning of the human brain, neuroscience has remained distant from classrooms. Several scholars have argued that this disconnection has genuine intellectual and practical roots. In a celebrated article, John Bruer described the relationship between brain research and education as a 'bridge too far' ${ }^{1}$ and argued that pertinence to neuroscience and to education need not agree. For instance, it may be very relevant to neuroscience that parietal and prefrontal regions are key nodes for number processing ${ }^{2}$, but this does not seem to help a teacher teach math.

The bridge too far argument assumes a linear flow from neuroscience to cognitive psychology and education. We argue instead

${ }^{1}$ Laboratorio de Neurociencia Integrativa, Physics Department, FCEyN, Universidad de Buenos Aires and IFIBA, CONICET, Buenos Aires, Argentina. ${ }^{2}$ Universidad Torcuato Di Tella, Buenos Aires, Argentina. ${ }^{3}$ Laboratorio de Neurociencias Cognitivas, Escuela de Psicología, Pontificia Universidad Católica de Chile. ${ }^{4}$ Instituto do Cérebro, Universidade Federal do Rio Grande do Norte (UFRN), Natal, Brazil. Correspondence should be addressed to M.S. (sigman@df.uba.ar).

Received 4 September 2013; accepted 5 February 2014; published online 26 March 2014; doi:10.1038/nn.3672 that neuroscience and cognitive psychology should work in synergy, providing complementary tools to understand the mind and act in concert to improve education.

There is a long history of translating knowledge from basic to applied science. One view is that science should produce a vast corpus of basic knowledge, hoping that some of it will eventually be useful for societal needs. An alternative approach, coined by Donald Stokes as the Pasteur Quadrant ${ }^{3}$, is to find a niche in which applied and basic research are equally pertinent. In Stokes' taxonomy, scientific knowledge is classified according to whether it constitutes a quest for fundamental understanding and whether it has considerations of use. Bohr's model of the atom constitutes an example of science pursued by the pure sake of knowledge, whereas Edison's light bulb is an example of a pure drive for considerations of use. Pasteur's research on vaccination excels in both dimensions, at the same time solving fundamental principles of microbiology and providing a solution to one of the most dramatic medical problems of the era. Here we critically examine four domains in which neuroscience, cognitive psychology and education meet at the Pasteur Quadrant to simultaneously answer fundamental theoretical questions of brain function and improve the quality and effectiveness of educational practices.

\section{Physiology of education: for starters, a good morning}

Students in most countries of the world attend school experiencing the physiological effects of a poor diet. The brain is by far the largest consumer of glucose among all body organs, and glucose administration before training boosts short-term as well as long-term learning 4 . This means that, irrespective of the pedagogical methods employed, inadequate nutrition, which is most prominent in students with low socioeconomic status, will hamper learning ${ }^{5}$. Caloric intake is not the only dietary requirement for learning; a high-fat diet leads to the desensitization of NMDA receptors that are critical for learning 6 . The effort to fully understand the relationship between meal composition and learning should be taken as a serious challenge by neuroscientists, as nutritional interventions can effectively improve educational outcomes. Provision of an adequate school breakfast produces benefits in 
children's nutritional status, which cascades to higher school attendance and achievement, specifically improving performance in arithmetic ${ }^{7}$. Comparable results were observed in Rural China's Elementary Schools providing children with multivitamin supplementation ${ }^{8}$.

Also ripe is the need for a systematic investigation of optimal exercise schedules in school, given the overwhelming evidence that physical exercise benefits cognition through a variety of mechanisms that likely include upregulation of neurotrophic factors and enhancement of hippocampal neurogenesis and size ${ }^{9}$. Notably, exercise and cognition are synergic: although aerobic activity alone leads to an increase in capillary density and neurogenesis, enhanced astrocyte and neuropil volumes only occur when aerobic exercise is combined with environmental enrichment. Furthermore, persistent changes in neurogenesis and neuropil volume, beyond the period of exercise, require environmental enrichment ${ }^{10}$.

Although the parallel between enriched education and environmental enrichment is tempting, it must be emphasized that the literature on animals shows that massive stimulus deprivation is debilitating and can be restored by giving the animal a more normal environment. However, these studies do not show the benefits of additional stimulation as compared with 'normal' or moderately low levels of stimulation, which would be more pertinent to typical educational practices. The work on enriched environment in animals may therefore be most relevant to special populations, such as children raised in orphanages. In fact, studies in such populations have consistently shown very severe cognitive and brain development deficits related to major decreases in individual attention, cognitive stimulation and emotional affection. Adopted children show a graded recovery of cognitive function that decreases with the age of adoption (see ref. 11 for an extensive review).

Among the physiological requirements for learning, sleep is one of the most promising game-changers for school education in the near future. Sleepiness in the classroom is considered to be the teachers' enemy, observed in even the youngest school-aged children. In addition, a large body of knowledge has accumulated that shows that sleep enhances memory processing and, conversely, that sleep disruption can result in major learning deficits ${ }^{12,13}$. In humans, sleep after memory acquisition has been shown to benefit the consolidation ${ }^{14}$, restructuring ${ }^{15}$, generalization and selective remembering of memories ${ }^{16}$. These psychological discoveries have been paralleled by human imaging studies showing that the reactivation and neuroanatomical reorganization of memory traces are favored by sleep and proportional to learning ${ }^{12}$.

The search for mechanisms underlying the cognitive role of sleep showed that slow oscillations typical of non-rapid eye movement (NREM) sleep are augmented after learning ${ }^{17}$, are proportional to the amount of learning in preschoolers ${ }^{18}$ and can increase learning when experimentally boosted ${ }^{19}$. These findings close a causal loop that relates mnemonic gain to early-sleep neural synchronization. Animal studies have demonstrated that sleep triggers mnemonic reverberation at the level of action potentials ${ }^{20}$ as well as calcium-dependent molecular events related to long-term memory storage ${ }^{21,22}$.

One apparently trivial change that might capitalize on these findings suggesting the importance of sleep would be to delay the time of school onset. Students typically arrive sleepy for morning classes. The physiological inertia of sleep states that lingers during waking and the major changes in sleep regulation during adolescence ${ }^{23}$ may explain why so many teenagers are ill prepared for learning early in the morning 24 .

Starting school later ${ }^{25}$ is a relatively easy implementation that could enhance learning, but is unpopular among parents, who need to go to work around the same time school starts. Rather than asking students to refrain from sleeping at school or to arrive later, naps could be explicitly employed as educational tools. Naps restore cognitive fatigue and, for certain tasks, provide performance gains comparable to those obtained after a full night of sleep ${ }^{26}$. In adults, different sleep phases have specific effects on distinct kinds of memories: whereas NREM sleep is crucial for the consolidation of declarative memories, REM sleep is required for the consolidation of procedural memories $^{12}$. In children, however, sleep-dependent benefits have only been detected for declarative memories ${ }^{13}$. This is fortunate for the purpose of applying naps to school learning, as most content learned in school involves declarative memories and the bulk of diurnal naps involves NREM sleep ${ }^{12}$. Neuroscience research suggests that sleep, nutrition and exercise influence learning, and therefore constitute the physiological foundation of pedagogy.

\section{Education outside the school: language acquisition}

People usually identify education and schooling as one and the same. However, considering school hours, weekends, holydays and vacations, children spend $85 \%$ of their waking time outside of school. Parent-child relationships have a major role in child development on their own, as well as through interactions with formal education ${ }^{27,28}$. Cognitive psychology has identified other relevant factors for learning outside the classroom: the amount and quality of play, and emotional and regulatory mechanisms that influence temperament and school readiness ${ }^{29,30}$. Traits trained during early development may cascade over subsequent school competences ${ }^{27}$.

Neuroscience research has developed signatures that may serve to diagnose cognitive impairments potentially earlier than would be conceivable by behavioral or psychological inspection. A paradigmatic example is the detection of otoacoustic emissions in neonates, a tool that helps identify congenital deafness. Traditional detection by psychological tests can only be made months after birth, missing a window of opportunity for early interventions ${ }^{31}$. A large case cohort analysis of 41,796 children born in Colorado (between 1992 and 1996) demonstrated that universal hearing screening of newborns is highly beneficial, from an economical perspective, considering the recovery of all screening costs in the prevention of future intervention ${ }^{32}$. Notably, early intervention made possible by newborn screenings has been related to significant improvement in the academic performance of deaf peo$\mathrm{ple}^{33,34}$. Children receiving cochlear implants before 3 years of age show faster rates of speech and vocabulary production when compared with age-matched peers who had received their implants at later ages ${ }^{35}$; earlier implantations also lead to improved reading comprehension ${ }^{36}$.

The infant's mind had long been conceived as a tabula rasa, void of all conceptual content and gradually filled by accumulating sensory experience. Today, however, there is ample evidence demonstrating that infants possess a core of conceptual knowledge that includes notions of mathematics, social cognition and language ${ }^{37}$. Although most evidence comes from behavioral studies, various aspects of infant thought are encapsulated in the mind, unexpressed by any motor effector ${ }^{38}$. Capitalizing on neuroscience tools to infer the mental content of infants and young children is particularly relevant in the study of preverbal language development. Studies of brain activity evoked by normal and reversed speech in awake and sleeping 2-d-old infants showed that left-lateralized brain regions similar to those of adults, including the superior temporal and angular gyri, are already active in infants ${ }^{39}$. High-temporal resolution functional magnetic resonance imaging revealed fast responses in preverbal infants in the vicinity of the primary auditory cortex, whereas responses became increasingly slower toward the posterior part of the superior temporal gyrus and toward the temporal poles and inferior frontal regions (Broca's area $)^{40}$. Thus, pre-linguistic infants show brain activity that is consistent with a rudimentary form of language. 
Gaining awareness of covert aspects of the infant's mind may help parents improve the quality of their interaction with their children. Parents naturally assist their infants on their primordial tasks, such as reaching, grasping, crawling, walking, etc. A key element for such pedagogic drive is to recognize the child's intentions. If parents knew that 3-month-old infants were likely to produce a rudimentary form of language that is not routed to motor action, they could gain motivation to be more conversational with their children, training the developing language system in the same way that a parent gives a hand to his child when the child's intention to begin walking becomes apparent.

Another key element of pedagogy is to understand what the pupil is prepared to do at each stage. No parent would ask a 6-month-old child to run, much less be frustrated if this does not happen. As absurd as this example sounds, comparable situations often proceed unnoticed in the domain of cognition. Parents might avoid demanding the impossible of their children if they knew of Michael Posner's work on the development of attentional networks ${ }^{41}$, which suggests that young infants can orient (direct attention) to a new item, but may not have yet developed a system to disengage, that is, voluntarily remove attention from an attended object or behavior. Similarly, it could help parents to understand that the slow and progressive development of control mechanisms during childhood ${ }^{42}$ makes the consideration of future rewards much less likely in young children ${ }^{43}$. This could help parents to focus on immediate consequences when negotiating with their young children.

\section{Neuroscience, norms and ideology: the case of bilingualism}

Neuroscience and cognitive psychology have recently assumed a leading role in elucidating whether simultaneous exposure to multiple languages confuses or stimulates children. This heated discussion occurs in heavily charged political contexts, such as immigration in the USA or cultural segregation in Europe. It is then often tempting to rapidly convert scattered neuroscience findings into educational dogmas that glorify or demonize the cognitive effects of bilingualism.

Scientific and nonscientific discourses typically consider monolingualism as the norm ${ }^{44}$, and the performance of bilinguals is perceived as a deficit or an enhancement relative to that norm ${ }^{45}$. This is not a mere convention: as discussed below, bilingual children have an advantage in executive functions, but this is never perceived as monolinguals experiencing a deficit in this domain. Notably, the monolingual norm is not set by ubiquity; in fact most children in the world grow up in multilingual environments ${ }^{46}$.

The differences in cognitive and brain development between bilinguals and monolinguals have been extensively reviewed elsewhere ${ }^{45,47}$. Here we focus on a few aspects that are specifically pertinent to education. Research has been quite conclusive in establishing that, contrary to popular belief, the important milestones of language acquisition are similar among monolinguals and bilinguals ${ }^{48}$. The most prominent and consistently documented advantage of bilingual children is in executive functions, more specifically in tasks requiring inhibition of attention, monitoring and cognitive control ${ }^{47,49}$. Other comparisons instead may show an advantage for monolinguals. An investigation of a large sample of 1,738 children showed that monolinguals had significantly larger vocabularies than bilinguals at every age examined, from 3 to 10 years of age. However, this effect vanishes when vocabulary is aggregated for bilinguals across both languages ${ }^{50}$. Bilingualism also consistently causes a linguistic disadvantage in lexical access even for bilinguals' first and dominant language ${ }^{51}$.

Neuroscience research can help to elucidate the brain mechanisms that allow bilinguals to achieve better executive functions scores.
Switching between several tasks (a classic probe of executive functions) correlates with the activation of language networks in bilinguals (but not monolinguals) even in non-linguistic tasks ${ }^{52}$. In conflict tasks that involve monitoring, bilinguals show less activation of the anterior cingulate cortex ${ }^{53}$, a fundamental hub for the coordination of cognitive control ${ }^{54}$. These findings add up to an appealing idea: bilinguals scaffold cognitive control by recycling structures that in monolinguals are, to a great degree, specialized for language. Bilinguals have greater white matter density in the anterior cingulate than monolinguals ${ }^{53}$, indicating that the language-driven reorganization of attentional networks with bilingualism also has anatomical consequences.

Anatomical differences between monolinguals and bilinguals can be long-lasting. Bilingualism is associated with a delay of dementia symptoms in the elderly ${ }^{55}$ and it has been conjectured that the correlation of lifelong bilingualism with white matter integrity may be at the root of increased cognitive reserve ${ }^{56}$. Although this idea is still incipient, it presents an interesting example of how neuroscience and education need not be constrained to early development, and may instead be pertinent throughout the entire life span.

Cognitive control during childhood is a strong predictor of school and social competence ${ }^{57}$, which seems to be a good argument to stimulate bilingualism. However, the reduction in vocabulary and lexical access may have a negative effect on academic performance. How are these cognitive differences in performance believed to affect educational achievement? Even if research on bilingualism has flourished over the last years, current evidence may not yet be sufficiently consolidated to convey a decisive response to this question ${ }^{45}$. The main argument is that one cannot conclude that variations in performance in any given task should promote a societal, educational or parental reaction when the causes and consequences of such variations in real-world measures are not yet understood ${ }^{58}$. Bilingualism is a good example of why neuroscientists should be prudent when communicating their findings to the general public.

\section{Perceptual learning: natural scenes statistics to reading}

Reading, math and other cognitive skills taught in schools present a paradox for neuroscience simply because not enough time has passed to allow the evolution of specific neural circuitries for these cultural traits. At birth, our brains are likely identical to those of our illiterate ancestors 5,300 years ago, when the first written records were made. A possible solution to this conundrum is the neuronal recycling hypothesis ${ }^{59}$, built on Stephen Jay Gould's notion of exaptation ${ }^{60}$. It posits that new cultural acquisitions (tools, music, reading and math) are only possible when they fit into a preexisting neural architecture developed for more fundamental functions ${ }^{59}$. This theory has a logical implication: if reading is instantiated in ancient cortical circuits for vision, the architecture of these circuits must shape and constrain the way that we read.

The fundamental architectonic bricks of the primary visual cortex (V1), such as ocular dominance columns, can only change in response to experience during a limited critical period, restricted to the first few months of life ${ }^{61}$. In the early days of neurophysiology, this finding was extrapolated to infer that cortical plasticity of all sorts was severely dampened during adulthood. This view was hegemonic for many years, but changed radically over the last two decades. There is now a consensus that broad forms of perceptual learning and cortical plasticity occur throughout the human life $\operatorname{span}^{62}$.

Literacy is perhaps the most remarkable perceptual-learning experience in modern societies ${ }^{63}$. It is a radical transformation, after which a set of visual symbols becomes automatically mapped to auditory phonemes. This, in turn, has a cascading effect on cognition because 
literacy scaffolds a myriad of other aspects of human culture. It seems quite reasonable then that the neuroscience of perceptual learning, a flourishing literature in the brain sciences, should be connected to the reading literature ${ }^{64}$.

Three complementary sources of evidence suggest that words are the units of reading. First, eye movements during fluent reading are made mostly by making saccades from one word to the next ${ }^{65}$. Second, the reading time of a single word is relatively independent of the number of letters ${ }^{66}$. Third, a single letter may be more easily detected in brief presentations when embedded in a word ${ }^{67}$. A possible inference of these findings is that education should be organized to teach children to read entire words instead of focusing in letterby-letter identification. This procedure, usually termed holistic reading, led to concrete implementations that turned out to be a major pedagogical fiasco ${ }^{68}$.

As it turns out, the neuroscience of visual learning could actually have predicted this failure. The development of literacy is a case of pop-out learning, a process by which, after extensive practice, one can identify a specific set of shapes in cluttered fields very rapidly and with a subjective feeling of automaticity and lack of effort. For nonreaders, reading is a slow, effortful and serial process that becomes automatic after many hours of training ${ }^{66}$. What sort of transformation elicits this type of learning in the brain and what material is optimal for this learning process?

Constitutive elements of shapes are represented by pools of neurons encoding basic traits (strokes) that recombine to form new elements of intermediate complexity, which are subsequently recombined to encode more complex objects ${ }^{69}$. This notion was incorporated into a model of neural codes for written words, based on a hierarchy of increasingly complex neuronal detectors, from individual letters to bigrams and morphemes. Only specific patterns that conform certain letters from strokes (as opposed to other patterns with similar regularities, but which do not occur in the alphabet) are trained by visual experience ${ }^{64}$. The hypothesis was that this process relies on the same learning mechanisms that carve a cortical circuitry for grouping contours and segmenting textures, namely the assembling of object statistical regularities in the visual world ${ }^{62}$. This hypothesis was tested by measuring brain responses to visual strings that progressively disrupt the 'natural statistics' of the alphabet at different scales: JZWYZK (infrequent letters), QOADTM (frequent letters), QUMBST (frequent bigrams) and AVONIL (frequent quadrigrams). Results showed a gradient of selectivity spanning the left occipitotemporal cortex, with increasing selectivity for higher level stimuli toward the anterior fusiform region ${ }^{70}$.

The importance of this finding for education is that even after extensive practice with reading, words are still represented by their constitutive components. This process goes all the way to what appears to be the constitutive elements of all alphabets, that is, oriented elements or strokes. For this reason, one aspect that may impair fluent reading is the inability to parse words into letters. In agreement with this prediction, the remarkably simple intervention of increasing letter spacing substantially improves text reading in some kinds of dyslexic children ${ }^{71}$. An additional piece of evidence required to bring these data together is that visual crowding, the inability to identify objects in clutter, is more severe in dyslexic children, making it hard to parse letters from continuous words ${ }^{72}$.

An even more direct and concrete effect of neuroscience in reading can be found in interventions in children with deficits in phonological processing, the most frequent cause of dyslexia in English speakers ${ }^{73}$. These children have difficulty performing phonological awareness tasks such as knowing what word remains if the sound ' $\mathrm{P}$ ' is deleted from the word 'Paris'73. Brain research shows that, when performing these kinds of tasks, dyslexic children show hypo-activation in the left temporo-parietal cortex, which is believed to link auditory and visual processes during reading ${ }^{74}$. How could this finding help a teacher deal with a dyslexic child?

The diagnosis of dyslexia is typically made in children aged 7-8 years old, when population variability in reading scores becomes evident. However, interventions to remediate dyslexia are much more likely to be successful when conducted on children who are beginning to read or even before reading if they are based exclusively on improving auditory processing ${ }^{75}$. As with many other medical conditions, early diagnosis is a fundamental aspect of remediation. The development of neurophysiological markers of later dyslexic development are therefore of great practical relevance. A concrete example, reminiscent of the otoacoustic emission study described above, is the event-related potentials (ERPs) to language sounds. For instance, the Jyväskylä Longitudinal Study of Dyslexia found that, as early as birth, infants with and without familial risk for dyslexia differ in ERPs to linguistic stimuli. These differences persist in pre-school and school-age children ${ }^{76}$. Notably, early ERP measures are predictive of reading skills at school ${ }^{77,78}$. Another study showed that ERPs obtained hours after birth have a strong relation to reading skills many years later, predicting whether the infant will develop dyslexia at age 8 with $81 \%$ accuracy ${ }^{79}$. Taken together, these studies indicate that ERPs measured during infancy might help to screen for problems in reading-related skills, serving as an indicator of risk of impaired auditory/speech processing. A cautionary note is necessary: ERPs are highly variable in infants and, when conceiving concrete recommendations, it is important to distinguish inferences that derive from a single study with a relatively low sample size from studies performed on large samples or with several independent replications.

\section{Getting practical: the ethics of school experimentation}

Scientific facts are nearly always constructed by isolating the studied factor from other variables. However, for educational relevance, one has to ask questions in a broader context, considering ethical, economic and social implications. For instance, laboratory research has shown that sleep benefits memory consolidation. In school, one has to judge whether the gain potentially induced by the use of sleep compensates for the lack of time devoted to classes. Can sleep in a room shared with other students be as efficient for learning as sleep in a quiet laboratory room? How do naps interact with other variables such as nutrition, exercise and levels of intrinsic motivation?

Thus, when asking how neuroscience can be useful to education it is insufficient to focus solely on our current understanding of brain function. Efforts to make change may be wasted if they are not accompanied by a reflection on how the translational process can be efficiently organized. Neuroscientists should be aware that neuroscience is a fashionable field with increasing commercial appeal and that they therefore have a responsibility to disseminate a critical, rigorous view of neuroscience results so as to firmly support the dialog with nonscientists. Here we summarize what we think are five constitutive pillars to optimize this dialog.

First, educational neuroscience must shape the practical and ethical aspects of linking the biology of learning and memory to human formal education, taking care of recommendations on what, how, when and where neuroscience can or cannot be relevant to education. Second, field studies to examine the validity of neuroscience theories in the classroom constitute a nearly unexploited research frontier that is crucial to prevent teachers, principals and decision makers (who are not experts in neuroscience) from arbitrarily picking, from the vast 
and heterogeneous body of empirical findings, solely concepts useful for their purposes. Third, education should be a source of inspiration for neuroscience research, contributing unique problems and novel experimental conditions. In particular, educational neuroscience should integrate teachers' knowledge to advance into more ecological experimental designs that may help scientists understand pedagogical practice and how the classroom may serve as experimental locus. Fourth, fundamental brain concepts should be part of the teacher's professional program so as to provide a scientific tool to deal with myths and prejudice. Fifth, as in other interdisciplinary enterprises, a crucial investment for success will be to promote a new generation of students capable of progressing in the investigation of the links between education, cognition and brain function.

Now is the time to be both practical and brave, identifying the most promising findings provided by neuroscience, and using them to design and implement transformative educational experiments. Current work on the construction of direct links between brain data and pedagogical interventions promises to be a particularly important field of research for future neuroscience, as Hebb anticipated more than 60 years ago.

\section{ACKNOWLEDGMENTS}

The authors thank the faculty and students of the Latin American School for Education, Cognitive and Neural Sciences (LA School) for kindling the ideas presented here, the James S. McDonnell Foundation for supporting the LA School, D. Koshiyama for librarian support, G. Gellon for reading and commenting the manuscript, and D. Klahr for inspiring the notion that educational neuroscience lies in Pasteur's Quadrant. S.R. is funded by grant 14385 (ACERTA), 049/2012/CAPES/INEP - Programa Observatório da Educação. M.S. and A.P.G. are funded by CONICET and UBACYT. M.S. is sponsored by a scholar award of the James McDonnell Foundation. M.P. is sponsored by CONICYT Chile, Fondecyt \# 1110928 and IDeA CA12I10372.

\section{COMPETING FINANCIAL INTERESTS}

The authors declare no competing financial interests.

Reprints and permissions information is available online at http://www.nature.com/ reprints/index.html.

1. Bruer, J.T. Education and the Brain: A bridge too far. Educ. Res. 26, 4-16 (1997).

2. Arsalidou, M. \& Taylor, M.J. Is $2+2=4$ ? Meta-analyses of brain areas needed for numbers and calculations. Neuroimage 54, 2382-2393 (2011).

3. Stokes, D.E. Pasteur's Quadrant: Basic Science and Technological Innovation (Brookings Institution Press, 1997).

4. Korol, D.L. \& Gold, P.E. Glucose, memory, and aging. Am. J. Clin. Nutr. 67, 764S-771S (1998).

5. Hackman, D.A., Farah, M.J. \& Meaney, M.J. Socioeconomic status and the brain: mechanistic insights from human and animal research. Nat. Rev. Neurosci. 11, 651-659 (2010)

6. Valladolid-Acebes, I. et al. High-fat diets induce changes in hippocampal glutamate metabolism and neurotransmission. Am. J. Physiol. Endocrinol. Metab. 302, E396-E402 (2012).

7. Grantham-McGregor, S. Can the provision of breakfast benefit school performance? Food Nutr. Bull. 26, S144-S158 (2005).

8. Renfu, L. et al. Nutrition and educational performance in rural China's elementary schools: results of a randomized control trial in Shaanxi Province. Econ. Dev. Cult. Change 60, 735-772 (2012).

9. Erickson, K.I. et al. Exercise training increases size of hippocampus and improves memory. Proc. Natl. Acad. Sci. USA 108, 3017-3022 (2011).

10. Van der Borght, K. et al. Physical exercise leads to rapid adaptations in hippocampal vasculature: temporal dynamics and relationship to cell proliferation and neurogenesis. Hippocampus 19, 928-936 (2009).

11. Perry, B.D. Childhood experience and the expression of genetic potential: What childhood neglect tells us about nature and nurture. Brain Mind 3, 79-100 (2002).

12. Diekelmann, S. \& Born, J. The memory function of sleep. Nat. Rev. Neurosci. 11, 114-126 (2010)

13. Wilhelm, I., Diekelmann, S. \& Born, J. Sleep in children improves memory performance on declarative but not procedural tasks. Learn. Mem. 15, 373-377 (2008).

14. Stickgold, R., James, L. \& Hobson, J.A. Visual discrimination learning requires sleep after training. Nat. Neurosci. 3, 1237-1238 (2000).
15. Wagner, U., Gais, S., Haider, H., Verleger, R. \& Born, J. Sleep inspires insight. Nature 427, 352-355 (2004).

16. Wilhelm, I. et al. Sleep selectively enhances memory expected to be of future relevance. J. Neurosci. 31, 1563-1569 (2011).

17. Huber, R., Ghilardi, M.F., Massimini, M. \& Tononi, G. Local sleep and learning. Nature 430, 78-81 (2004).

18. Kurdziel, L., Duclos, K. \& Spencer, R.M.C. Sleep spindles in midday naps enhance learning in preschool children. Proc. Natl. Acad. Sci. USA 110, 17267-17272 (2013).

19. Marshall, L., Helgadóttir, H., Mölle, M. \& Born, J. Boosting slow oscillations during sleep potentiates memory. Nature 444, 610-613 (2006).

20. Wilson, M. \& McNaughton, B. Reactivation of hippocampal ensemble memories during sleep. Science 265, 676-679 (1994).

21. Ribeiro, S. et al. Induction of hippocampal long-term potentiation during waking leads to increased extrahippocampal zif-268 expression during ensuing rapid-eyemovement sleep. J. Neurosci. 22, 10914-10923 (2002).

22. Vecsey, C.G. et al. Sleep deprivation impairs cAMP signaling in the hippocampus. Nature 461, 1122-1125 (2009).

23. Hagenauer, M.H., Perryman, J.I., Lee, T.M. \& Carskadon, M.a. Adolescent changes in the homeostatic and circadian regulation of sleep. Dev. Neurosci. 31, 276-284 (2009).

24. Hansen, M., Janssen, I., Schiff, A., Zee, P.C. \& Dubocovich, M.L. The impact of school daily schedule on adolescent sleep. Pediatrics 115, 1555-1561 (2005).

25. Owens, J.A., Belon, K. \& Moss, P. Impact of delaying school start time on adolescent sleep, mood, and behavior. Arch. Pediatr. Adolesc. Med. 164, 608-614 (2010).

26. Mednick, S., Nakayama, K. \& Stickgold, R. Sleep-dependent learning: a nap is as good as a night. Nat. Neurosci. 6, 697-698 (2003).

27. Melhuish, E.C., Sylva, K. \& Sammons, P. Preschool influences on mathematics achievement. Science 321, 1161-1162 (2008).

28. Farah, M.J. et al. Environmental stimulation, parental nurturance and cognitive development in humans. Dev. Sci. 11, 793-801 (2008).

29. Fisher, K., Hirsh-pasek, K., Golinkoff, R.M., Singer, D.G. \& Berk, L. Playing around in school: implications for learning and educational policy in Oxford Handb. Dev. Play (Pellegrini, A.) 341-362 (Oxford University Press, 2011).

30. Rothbart, M.K. \& Jones, L.B. Temperament, self-regulation and education. School Psych. Rev. 27, 479-491 (1998).

31. Korver, A.M.H. et al. Newborn hearing screening vs later hearing screening and developmental outcomes in children with permanent childhood hearing impairment. J. Am. Med. Assoc. 304, 1701-1708 (2010).

32. Mehl, A.L. \& Thomson, V. Newborn hearing screening: the great omission. Pediatrics 101, e4 (1998).

33. Francis, H.W. \& Niparko, J.K. Cochlear implantation update. Pediatr. Clin. North Am. 50, 341-361 (2003).

34. Semenov, Y.R., Martinez-Monedero, R. \& Niparko, J.K. Cochlear implants: clinical and societal outcomes. Otolaryngol. Clin. North Am. 45, 959-981 (2012).

35. Connor, C.M., Craig, H.K., Raudenbush, S.W., Heavner, K. \& Zwolan, T.A. The age at which young deaf children receive cochlear implants and their vocabulary and speech-production growth: is there an added value for early implantation? Ear Hear. 27, 628-644 (2006)

36. Connor, C.M. \& Zwolan, T.A. Examining multiple sources of influence on the reading comprehension skills of children who use cochlear implants. J. Speech Lang. Hear. Res. 47, 509-526 (2004).

37. Carey, S. The Origin of Concepts (Oxford University Press, 2009).

38. Evers, K. \& Sigman, M. Possibilities and limits of mind-reading: a neurophilosophical perspective. Conscious. Cogn. 22, 887-897 (2013).

39. Peña, M. et al. Sounds and silence: an optical topography study of language recognition at birth. Proc. Natl. Acad. Sci. USA 100, 11702-11705 (2003).

40. Dehaene-Lambertz, G. et al. Functional organization of perisylvian activation during presentation of sentences in preverbal infants. Proc. Natl. Acad. Sci. USA 103 14240-14245 (2006).

41. Petersen, S.E. \& Posner, M.I. The attention system of the human brain: 20 years after. Annu. Rev. Neurosci. 35, 73-89 (2012).

42. Davidson, M.C., Amso, D., Anderson, L.C. \& Diamond, A. Development of cognitive control and executive functions from 4 to 13 years: evidence from manipulations of memory, inhibition and task switching. Neuropsychologia 44, 2037-2078 (2006).

43. Atance, C.M. \& O'Neill, D.K. Episodic future thinking. Trends Cogn. Sci. 5, 533-539 (2001).

44. Medin, D., Bennis, W. \& Chandler, M. Culture and the home-field disadvantage. Perspect. Psychol. Sci. 5, 708-713 (2010).

45. Akhtar, N. \& Menjivar, J.A. Cognitive and linguistic correlates of early exposure to more than one language. Adv. Child Dev. Behav. 42, 41-78 (2012).

46. UNESCO. Education in a multilingual world. <http://unesdoc.unesco.org/images/ 0012/001297/129728e.pdf>. (2003).

47. Bialystok, E. Bilingualism: The good, the bad, and the indifferent. Biling. Lang. Cogn. 12, 3-11 (2009).

48. Werker, J.F. \& Byers-Heinlein, K. Bilingualism in infancy: first steps in perception and comprehension. Trends Cogn. Sci. 12, 144-151 (2008).

49. Kovács, A.M. \& Mehler, J. Cognitive gains in 7-month-old bilingual infants. Proc. Natl. Acad. Sci. USA 106, 6556-6560 (2009).

50. Bialystok, E., Luk, G., Peets, K.F. \& Yang, S. Receptive vocabulary differences in monolingual and bilingual children. Lang. Cogn. 13, 525-531 (2010).

51. Ivanova, I. \& Costa, A. Does bilingualism hamper lexical access in speech production? Acta Psychol. (Amst.) 127, 277-288 (2008). 


\section{REVIEW}

52. Garbin, G. et al. Bridging language and attention: brain basis of the impact of bilingualism on cognitive control. Neuroimage 53, 1272-1278 (2010).

53. Abutalebi, J. et al. Bilingualism tunes the anterior cingulate cortex for conflict monitoring. Cereb. Cortex 22, 2076-2086 (2012).

54. Posner, M.I., Rothbart, M.K., Sheese, B.E. \& Tang, Y. The anterior cingulate gyrus and the mechanism of self-regulation. Cogn. Affect. Behav. Neurosci. 7, 391-395 (2007).

55. Bialystok, E., Craik, F.I.M. \& Freedman, M. Bilingualism as a protection against the onset of symptoms of dementia. Neuropsychologia 45, 459-464 (2007).

56. Luk, G., Bialystok, E., Craik, F.I.M. \& Grady, C.L. Lifelong bilingualism maintains white matter integrity in older adults. J. Neurosci. 31, 16808-16813 (2011).

57. Mischel, W. et al. "Willpower" over the life span: decomposing self-regulation. Soc. Cogn. Affect. Neurosci. 6, 252-256 (2011).

58. Hoff, E. Interpreting the early language trajectories of children from low-SES and language minority homes: Implications for closing achievement gaps. Dev. Psychol. 49, 4-14 (2013).

59. Dehaene, S. \& Cohen, L. Cultural recycling of cortical maps. Neuron 56, 384-398 (2007).

60. Jay Gould, S. \& Vrba, E. Exaptation-a missing term in the science of form. Paleobiology 8, 4-15 (1982).

61. Wiesel, T.N. \& Hubel, D.H. Extent of recovery from the effects of visual deprivation in kittens. J. Neurophysiol. 28, 1060-1072 (1965).

62. Gilbert, C.D., Sigman, M. \& Crist, R.E. The neural basis of perceptual learning. Neuron 31, 681-697 (2001).

63. Carreiras, M. et al. An anatomical signature for literacy. Nature 461, 983-986 (2009).

64. Dehaene, S., Cohen, L., Sigman, M. \& Vinckier, F. The neural code for written words: a proposal. Trends Cogn. Sci. 9, 335-341 (2005).

65 . Rayner, K. Eye movements in reading and information processing: 20 years of research. Psychol. Bull. 124, 372-422 (1998).

66. Biemiller, A. Relationships between oral reading rates for letters, words, and simple text in the development of reading achievement. Read. Res. Q. 13, 223-253 (1977).
67. Reicher, G.M. Perceptual recognition as a function of meaninfulness of stimulus material. J. Exp. Psychol. 81, 275-280 (1969).

68. Dehaene, S. Reading in the Brain: the New Science of How We Read (Penguin books, 2009).

69. Ullman, S. Object recognition and segmentation by a fragment-based hierarchy Trends Cogn. Sci. 11, 58-64 (2007).

70. Vinckier, F. et al. Hierarchical coding of letter strings in the ventral stream dissecting the inner organization of the visual word-form system. Neuron 55, 143-156 (2007).

71. Zorzi, M. et al. Extra-large letter spacing improves reading in dyslexia. Proc. Natl. Acad. Sci. USA 109, 11455-11459 (2012).

72. Martelli, M. \& Di Filippo, G. Crowding, reading, and developmental dyslexia. J. Vis 9, 1-18 (2009).

73. Goswami, U. The development of reading across languages. Ann. NY Acad. Sci. 1145, 1-12 (2008)

74. Temple, E. et al. Neural deficits in children with dyslexia ameliorated by behavioral remediation: evidence from functional MRI. Proc. NatI. Acad. Sci. USA 100 2860-2865 (2003).

75. Gabrieli, J.D.E. Dyslexia: a new synergy between education and cognitive neuroscience. Science 325, 280-283 (2009).

76. Guttorm, T.K., Leppanen, P.H.T., Richardson, U. \& Lyytinen, H. Event-related potentials and consonant differentiation in newborns with familial risk for dyslexia. J. Learn. Disabil. 34, 534-544 (2001).

77. Guttorm, T.K., Leppänen, P.H.T., Hämäläinen, J.A., Eklund, K.M. \& Lyytinen, H.J. Newborn event-related potentials predict poorer pre-reading skills in children at risk for dyslexia. J. Learn. Disabil. 43, 391-401 (2010).

78. Leppänen, P.H. et al. Newborn brain event-related potentials revealing atypical processing of sound frequency and the subsequent association with later literacy skills in children with familial dyslexia. Cortex 46, 1362-1376 (2010).

79. Molfese, D.L. Predicting dyslexia at 8 years of age using neonatal brain responses. Brain Lang. 72, 238-245 (2000). 